\title{
Internal Benchmarking Improves Precision and Reduces Animal Requirements for Determination of Fish Bioconcentration Factors
}

\author{
Margaretha Adolfsson-Erici, ${ }^{\dagger}$ Gun Åkerman, ${ }^{\dagger}$ and Michael S. McLachlan*, ${ }^{\dagger}$ \\ ${ }^{\dagger}$ Department of Applied Environmental Science (ITM), Stockholm University, SE-106 91 Stockholm, Sweden \\ Supporting Information
}

ABSTRACT: The enactment of new chemical regulations has generated a large need for the measurement of the fish bioconcentration factor (BCF). Past experience shows that the $\mathrm{BCF}$ determination lacks precision, requires large numbers of fish, and is costly. A new protocol was tested that shortens the experiment from up to 12 weeks for existing protocols to 2 weeks and reduces the number of fish by a factor of 5 , while introducing internal benchmarking for the BCF determination. Rainbow trout were simultaneously exposed to 11 chemicals. The BCFs were quantified using one of the test chemicals, musk xylene, as a benchmark. These were compared with BCFs measured in a parallel

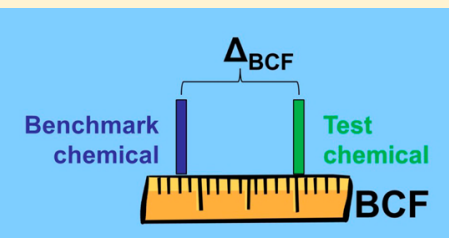
experiment based on the OECD 305 guideline. The agreement was $<20 \%$ for five chemicals and between $20 \%-25 \%$ for two further, while two chemicals lay outside the BCF operating window of the experiment and one was lost due to analytical difficulties. This agreement is better than that observed in a BCF Gold Standard Database. Internal benchmarking allows the improvement of the precision of BCF determination in parallel to large reduction in costs and fish requirements.

\section{INTRODUCTION}

The fish bioconcentration factor (BCF) is the quotient of a chemical's concentration in a fish and its concentration in water at steady state after the fish has been exposed to the chemical via water only. ${ }^{1}$ The fish $\mathrm{BCF}$ is an important metric in several chemical regulation frameworks, where it is used to evaluate the bioaccumulative properties of chemicals and for exposure assessment. The enactment of major chemical legislation in recent years has greatly increased the requirements for fish BCF testing. For example, the European chemicals management program $\mathrm{REACH}$ defines fish BCF thresholds as the primary criterion for deciding whether a chemical is classified as bioaccumulative or very bioaccumulative. Given the regulatory importance of this parameter and the large amount of testing required, it is important that the testing methodology is reproducible, economical, and efficient in its use of test animals.

The current standard method for the determination of fish BCF is the OECD guideline 305 "Bioconcentration: Flowthrough Fish Test” adopted in 1996. ${ }^{1}$ The experimental protocol prescribes an exposure phase lasting up to 4 weeks during which the test fish are exposed to the chemical in an aquarium, followed by a depuration phase lasting up to 8 weeks during which the fish are kept in chemical-free water. A minimum of 5 sampling points are prescribed during each phase, and at each sampling point a minimum of 4 fish are sacrificed and analyzed for their chemical residues. The guideline recommends that the experiment be conducted at two exposure levels. In summary, the method entails two experiments, each lasting up to 12 weeks, requiring the sacrifice and analysis of approximately 100 fish (including controls). This is costly, both economically and in its use of test animals. It has been estimated that the implementation phase of the European chemicals management program REACH will require approximately 65000 fish for BCF experiments. ${ }^{2}$ There is a need to find alternative methods that use fewer animals and are less costly. ${ }^{3}$ A first step was taken by Springer et al., who used statistic resampling of existing data from fish depuration experiments to demonstrate that contaminant elimination kinetics can be reliably quantified using fewer fish than are prescribed in the OECD 305 guideline. ${ }^{4}$ This has helped stimulate an ongoing revision of the OECD 305 guideline. $^{5}$ However, more needs to be done.

A fish bioconcentration study is difficult to conduct well, as it contains a range of experimental challenges and sources of variability. It requires holding fish growth and lipid constant within certain boundaries, maintaining constant chemical exposure conditions in the aquaria, and conducting precise analyses of the test chemicals in complex matrices. The BCF determination is based on a collection of measurements made in different fish instead of a single fish, so that variability between individual fish cannot be considered in calculating the BCF from the data.

The challenges in doing BCF experiments are reflected in poor reproducibility of many existing BCF measurements. This can be seen in the EURAS bioconcentration factor (BCF) Gold Standard Database (http://ambit.sourceforge.net/euras/), which has selected BCF values from the literature that satisfy rigorous quality assurance standards. Although $2 \mathrm{BCF}$ values are reported for most chemicals, these are largely from simultaneous studies at 2 different exposure concentrations. Therefore, reproducibility was assessed using those chemicals

Received: April 28, 2012

Revised: July 12, 2012

Accepted: July 18, 2012 
with more than 2 (maximum 6) reported BCF values. For chemicals which have a BCF between 500 and $20000 \mathrm{~L} \mathrm{~kg}^{-1}$ $w w$, the median quotient of the maximum and minimum lipidnormalized BCF value for a given chemical was 1.8. For all chemicals above the median the measurements had been done in the same fish species. This lack of precision in the BCF determination limits the utility of the experiments for evaluating chemicals against regulatory BCF thresholds.

In this study two hypotheses were tested. The first hypothesis was that it is possible to improve the precision of the OECD 305 guideline by using internal benchmarking. The internal benchmarking procedure entails simultaneously exposing the fish to a test chemical and to a chemical with a known BCF. The bioconcentration of the test chemical is then assessed relative to that of the benchmark chemical. The second hypothesis was that the length of the BCF experiments and the number of fish used could be greatly reduced without sacrificing the quality of the results if internal benchmarking was employed.

\section{THEORY}

The BCF of a contaminant in fish is defined as the quotient of the contaminant's concentration in fish $\left(C_{\mathrm{F}}\right)$ and its concentration at water $\left(C_{\mathrm{W}}\right)$ at steady state. In a BCF experiment the fish is exposed to the chemical via water only, not via diet.

The BCF can be determined by measuring $C_{\mathrm{F}}$ and $C_{\mathrm{W}}$ at steady state or by measuring $C_{\mathrm{F}}$ and $C_{\mathrm{W}}$ at nonsteady state and extrapolating to the steady state situation using a toxicokinetic model. The latter approach was used in this work. Typically the toxicokinetic model consists of a one compartment model of the fish and assumes first order kinetics for chemical exchange between the fish and water. This model is represented by the following differential equation

$$
\frac{\mathrm{d} C_{\mathrm{F}}}{\mathrm{d} t}=k_{1} C_{\mathrm{W}}-k_{2} C_{\mathrm{F}}
$$

where $C_{\mathrm{F}}$ is in $\mathrm{mol} \mathrm{kg}^{-1}, C_{\mathrm{W}}$ is in $\mathrm{mol} \mathrm{L} \mathrm{L}^{-1}, k_{1}$ is the uptake rate constant $\left(\mathrm{L} \mathrm{kg}^{-1} \mathrm{~d}^{-1}\right), k_{2}$ is the elimination rate constant $\left(\mathrm{d}^{-1}\right)$, and $t$ is time (d). At steady state this equation reduces to

$$
\mathrm{BCF}=\frac{C_{\mathrm{F}}}{C_{\mathrm{W}}}=\frac{k_{1}}{k_{2}}
$$

For lipophilic chemicals the BCF depends on the lipid content of the fish. To account for this and allow comparison of BCFs between individual fish, the BCF can be corrected to a standard lipid content of $5 \%$. This was done for this study.

The BCF can also be influenced by growth of the test organisms. When the fish grow, the concentration in the fish can decrease even if there is no elimination of chemical (i.e., $k_{2} C_{\mathrm{F}}$ in eq 1 is 0$)$. Growth can be included as a pseudoelimination process in the model, the definition of which then becomes

$$
\frac{\mathrm{d} C_{\mathrm{F}}}{\mathrm{d} t}=k_{1} C_{\mathrm{W}}-k_{2} C_{\mathrm{F}}-k_{\mathrm{G}} C_{\mathrm{F}}
$$

where $k_{\mathrm{G}}$ is the rate constant for growth $\left(\mathrm{d}^{-1}\right)$, i.e. the normalized rate of change of the fish's mass. The equation for the apparent $\mathrm{BCF}\left(\mathrm{BCF}_{\mathrm{A}}\right)$ becomes

$$
\mathrm{BCF}_{\mathrm{A}}=\frac{k_{1}}{k_{2}+k_{\mathrm{G}}}=\frac{k_{1}}{k_{\mathrm{T}}}
$$

where $k_{\mathrm{T}}$ is the rate constant for overall elimination including growth. When the magnitude of $k_{\mathrm{G}}$ is comparable to or greater than $k_{2}$, the BCF becomes dependent on the growth rate. Since $k_{2}$ typically decreases as BCF increases, the relevance of the growth rate for the $\mathrm{BCF}$ determination increases with the magnitude of $\mathrm{BCF}$. When $\mathrm{BCF}_{\mathrm{A}}$ is dependent on $k_{\mathrm{G}}$ (i.e., $\mathrm{BCF}_{\mathrm{A}}$ $\neq \mathrm{BCF})$, then it again becomes difficult to compare the measured $\mathrm{BCF}$ with the results of other experiments or to extrapolate the measured value to the field. This problem can be overcome by correcting the measured $\mathrm{BCF}$, i.e., converting $\mathrm{BCF}_{\mathrm{A}}$ back to $\mathrm{BCF}$ (from eqs 2 and 4)

$$
\mathrm{BCF}_{\mathrm{No}-\mathrm{G}}=\mathrm{BCF}_{\mathrm{A}} \times \frac{k_{2}+k_{\mathrm{G}}}{k_{2}}
$$

whereby we employ $\mathrm{BCF}_{\mathrm{No}-\mathrm{G}}$ instead of $\mathrm{BCF}$ to emphasize that this is a growth corrected $\mathrm{BCF}$ value. When $k_{2}$ becomes small compared to $k_{\mathrm{G}}$, then $\mathrm{BCF}_{\mathrm{A}}$ is entirely determined by the growth rate of the fish; it no longer contains quantitative information about the bioconcentration properties of the chemical.

\section{METHODS}

A modified experimental protocol, dubbed the BETTER-BCF (or BBCF) protocol, was developed. The structure of the OECD 305 guideline was retained, with an uptake phase followed by a depuration phase, but the length of the uptake phase was reduced to $4 \mathrm{~d}$ and the length of the depuration phase was reduced to $10 \mathrm{~d}$. Furthermore, the number of sampling points was reduced to one for the uptake phase and one for the depuration phase. This modified protocol was evaluated in an experiment with simultaneous exposure to 11 chemicals, one of which was employed as an internal benchmark. The results were compared with the results from a reference experiment conducted in parallel. The reference experiment was based on the OECD 305 guideline and studied the same chemicals in the same fish species. It has been published elsewhere. ${ }^{6}$ The differences between the experimental protocols for the BBCF and reference experiments are illustrated in Figure 1.

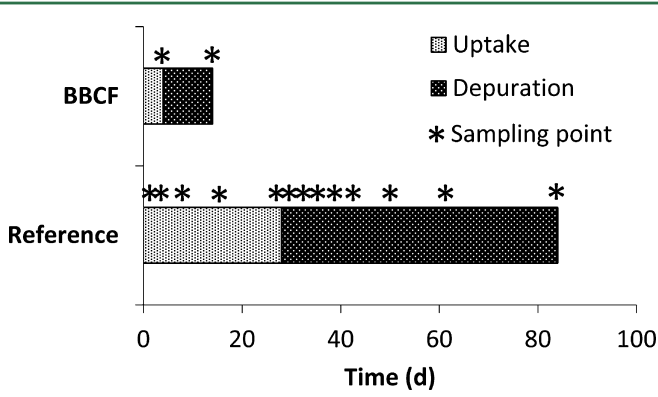

Figure 1. Schematic comparison of the sampling protocols for the $\mathrm{BBCF}$ experiment and the OECD 305-based reference experiment.

Chemicals. Ethyl acetate ('Pestiscan') was purchased from Labscan Ltd. Methanol ('LiChrosolv'), acetone ("SupraSolv"), and n-hexane ("LiChrosolv") were from Merck. Sodium chloride, phosphoric acid, and sulfuric acid were from $\mathrm{BDH}$. Silicone sheets (0.01" thickness) were from Specialty Silicone Products. SPE cartridges ( $1 \mathrm{~mL}$ of $50 \mathrm{mg}$ ISOLUTE ENV+) were from Biotage $A B$. The water was of milli-Q grade from a milli-Q ultrapure water system, Milli-Q PLUS 185 from Millipore. The chemicals to which the fish were exposed, the 
Table 1. List of the Test Chemicals and Their CAS Numbers and Abbreviations Together with the Overall Elimination Rate Constant $\left(k_{\mathrm{T}}\right)$, Uptake Rate Constant $\left(k_{1}\right)$, and Apparent Bioconcentration Factor $\left(\mathrm{BCF}_{\mathrm{A}}\right)$ for the $\mathrm{BBCF}$ Experiment, $\mathrm{BCF}_{\mathrm{A}}$ for the Reference Experiment, and Benchmarked $\mathrm{BCF}_{\mathrm{A}}$ for the BBCF Experiment

\begin{tabular}{|c|c|c|c|c|c|c|c|c|}
\hline \multirow[b]{2}{*}{ chemical } & \multirow[b]{2}{*}{ CAS no. } & \multirow[b]{2}{*}{ abbrev } & \multicolumn{2}{|c|}{$k_{\mathrm{T}}$} & \multirow[b]{2}{*}{$k_{1}\left(\mathrm{~L} \mathrm{~kg} \mathrm{lipid}{ }^{-1} \mathrm{~d}^{-1}\right)$} & \multicolumn{3}{|c|}{$\mathrm{BCF}_{\mathrm{A}}(\mathrm{L} \mathrm{kg} w w, 5 \%$ lipid $)$} \\
\hline & & & value $\left(d^{-1}\right)$ & $\overline{\operatorname{RSE}^{a}(\%)}$ & & $\mathrm{BBCF}$ & reference & BBCF benchmarked \\
\hline 2,6-diisopropyl-naphthalene ${ }^{b}$ & $24157-81-1$ & $\mathrm{DN}$ & 0.096 & 12 & & & 4500 & \\
\hline 2,3,4-trichloroanisole & $54135-80-7$ & ANI & 0.207 & 8 & 210 & 740 & 2036 & 1695 \\
\hline p-diisopropylbenzene & $100-18-5$ & ISBE & 0.358 & 12 & 144 & 294 & 801 & 672 \\
\hline musk xylene & $81-15-2$ & MUSK & 0.036 & 30 & 294 & 5928 & 13578 & 13578 \\
\hline chlorpyrifos & $2921-88-2$ & CHPY & 0.137 & 20 & 233 & 1235 & 3019 & 2828 \\
\hline 4-n-nonylphenol & $104-40-5$ & NP & 0.246 & 4 & 82 & 242 & 431 & 555 \\
\hline 2,4,6-tritert-butylphenol & $2051-60-7$ & BUFE & 0.004 & 245 & 238 & $43435^{c}$ & $30501^{c}$ & $99464^{c}$ \\
\hline pentachlorobenzene & $608-93-5$ & PENT & 0.030 & 37 & 423 & 10243 & 22943 & 23456 \\
\hline 2,5-dichlorobiphenyl & $34883-39-1$ & PCB & 0.028 & 45 & 1043 & 27330 & 80449 & 62586 \\
\hline hexachlorobenzene & $118-74-1$ & $\mathrm{HCB}$ & 0.020 & 56 & 441 & 15810 & 35727 & 36204 \\
\hline $\mathrm{p}, \mathrm{p}^{\prime}-\mathrm{DDT}$ & $50-29-3$ & DDT & 0.015 & 78 & 352 & $17028^{c}$ & $23665^{c}$ & $38994^{c}$ \\
\hline
\end{tabular}

${ }^{a} \mathrm{RSE}$ stands for relative standard error. ${ }^{b}$ The concentrations of DN in water were not determined, so $k_{1}$ could not be calculated. ${ }^{c} \mathrm{BCF}$ values not valid because the chemical lay outside of the BCF operating window of the experiment.

internal standards used for the analysis of these chemicals, and the sources of these chemicals are listed in Table S1 in the Supporting Information.

Fish/Aquarium. Juvenile rainbow trout were purchased from Näs Fiskodling $\mathrm{AB}$ at the age of 12 months and held in the laboratory's aquaria. They were 14 months old at the start of the experiment and weighed approximately 65-80 g. The fish were fed $3 \mathrm{~mm}$ pellets (EFICO Alpha from BioMar) once per day at a rate of $1 \%$ of their body weight. Fecal pellets were vacuumed from the bottom of the aquaria before feeding.

The experiments were conducted in $200 \mathrm{~L}$ aquaria made of fibreglass. The aquaria were circular with a central overflow drain. Charcoal filtered, aerated tap water was provided at a flow rate of $48 \mathrm{~L} \mathrm{~h}^{-1}$, giving an exchange rate of $5.8 \mathrm{~d}^{-1}$. An additional filter in the water supply removed particles down to $10 \mu \mathrm{m}$. The water in the aquaria was aerated. To prevent contamination of the laboratory the aquarium for the uptake experiment was covered with plastic, and a pump outfitted with an activated carbon filter was used to maintain an underpressure in the headspace. The temperature of the inflowing water and the aquaria room was maintained at $13{ }^{\circ} \mathrm{C}$.

Dosing System. Equilibrium passive dosing was used to generate a water supply with constant concentrations of the test chemicals during the uptake phase of the experiments. The method is described in detail elsewhere, ${ }^{7}$ and its application in this study is described in Adolfsson-Erici et al. ${ }^{6}$ It employed a PermSelect PDMSA-1.0 silicone membrane module (MedArray), which consisted of two manifolds connected by 16,128 hollow fibres made of polydimethyl siloxane (PDMS) with an inner diameter of $167 \mu \mathrm{m}$. The module was loaded with a methanol solution of the test chemicals, which was repeatedly perfused through the system while being progressively diluted with water. Thereafter, water was pumped through the module and the chemicals partitioned from the module's materials (PDMS and other) back into the water. The concentrations in water were independent of the water flow rate. ${ }^{7}$ Since the device operates by the partitioning principle, the concentrations generated are below saturation and can be regulated by varying the quantity of chemical loaded into the unit.

BBCF Experiment. Eight fish were placed in an aquarium that was being continuously supplied with water containing the test chemicals. Eleven chemicals which had been selected to cover a broad range of BCFs were tested simultaneously (see
Table 1 for the names of the chemicals and the abbreviations that will be used below). After $4 \mathrm{~d}$ four of the fish were sacrificed by severing the spine and stored at $-17{ }^{\circ} \mathrm{C}$ until analysis. The remaining four fish were transferred to an aquarium that was being continuously supplied with water that was free of the test chemicals. After a further $10 \mathrm{~d}$ these fish were also sacrificed and frozen. Ethical approval for the experiments was obtained from Stockholms Norra Djurförsöksetiska Nämnd (permit \# N 31/09).

Passive water samplers were deployed in the aquarium during the first two days of the uptake experiment. Silicone sheets (40 $\times 40 \times 0.25 \mathrm{~mm}$ ) were first cleaned by Soxhlet extraction in ethyl acetate for $6 \mathrm{~h}$ and dried. For sampling they were hung on a steel wire and submersed in the outflow region of the aquarium for $3 \mathrm{~h}$. On each day two samplers were deployed in parallel. Water samples (ca. $100 \mathrm{~mL}$ ) were collected in parallel with the passive sampler deployment. Sampling was done by immersing a $100 \mathrm{~mL}$ glass bottle in the aquarium close to the outflow.

Reference Experiment. The reference experiment was conducted in parallel to the BBCF experiment. It was based on the OECD 305 guideline and used the same methodologies employed for the BBCF experiment. The major differences were as follows: a) the exposure phase was $24 \mathrm{~d}$; b) there were 5 sampling points during the exposure phase (4 fish were sacrificed at each); c) passive water samplers were deployed on $6 \mathrm{~d}$ during the exposure phase; d) the depuration phase lasted $56 \mathrm{~d}$; and e) there were 8 sampling points during the depuration phase ( 4 fish were sacrificed at each). This study has already been reported elsewhere; ${ }^{6}$ in this paper the results will be compared with those from the BBCF experiment.

Analytical Methods. The samples were analyzed using the methods described previously. ${ }^{6}$ Briefly, the water samples were extracted on $50 \mathrm{mg}$ ENV+ SPE cartridges, followed by elution of the test chemicals in ethyl acetate. The silicone sheets were extracted by sonification in ethyl acetate for $30 \mathrm{~min}$ followed by volume reduction of the extract to $1 \mathrm{~mL}$. The fish were homogenized, extracted by shaking in n-hexane/acetone $(5: 1)$, re-extracted in pure $n$-hexane, washed with $9 \%$ sodium chloride in $1 \mathrm{M}$ phosphoric acid, treated with sulfuric acid, and then reduced in volume to $1 \mathrm{~mL}$. The extract was analyzed with GC/ MS using electron impact ionization. Quantification was performed using internal standards that were added to the 
sample after extraction. The lipid content of the fish was determined gravimetrically by evaporating an aliquot of the fish extract to dryness.

\section{RESULTS AND DISCUSSION}

No signs of ill health were observed for any of the fish during the experiment. At the time of sacrifice the fish weighed 66-84 $\mathrm{g}$ (mean $74 \mathrm{~g}$ ) and had a lipid content of 5.0\%-8.8\% (mean $6.9 \%$ ) (see Table S2).

Concentrations in Water $\left(C_{\mathrm{w}}\right)$. Only ANI, CHPY, and MUSK could be determined in the collected water, so the passive samplers were used to provide the concentrations in water. Preliminary experiments had shown that the silicone passive samplers did not approach equilibrium after a sampling time of $20 \mathrm{~h}^{6}$ As the samplers had been deployed for just $3 \mathrm{~h}$, the concentrations in the water were calculated assuming a constant sampling rate. This sampling rate was determined using the concentrations of ANI, CHPY, and MUSK in the water samples that had been collected in parallel. The average sampling rate for these three chemicals was used to calculate the concentration in the water from the concentration in the passive samplers. Details of the passive sampler calibration are given elsewhere. ${ }^{6}$

The concentrations in water ranged between $\sim 10($ PCB 9) and $\sim 1000 \mathrm{ng} \mathrm{L}^{-1}$ (musk xylene) (see Table S3). The median difference between the concentrations measured in the parallel samples was $5 \%$, indicating that the water analysis was precise. The median difference between the average concentrations measured on the two consecutive days was $15 \%$. The results from the reference experiment also showed that the passive dosing system maintains the concentrations of these test chemicals at relatively constant levels. ${ }^{6}$

Concentrations in Fish $\left(\boldsymbol{C}_{\text {Fish }}\right)$. The QA/QC parameters for the fish analysis method are documented elsewhere. ${ }^{8}$ Briefly, the recovery of the internal standards was $>80 \%$, and the repeatability of the analysis was $<4 \%$ for all of the analytes except 4-n-nonylphenol (13\%). Quantification limits were determined as five times the procedural blanks or five times the noise and ranged from 0.01 to $0.2 \mu \mathrm{g} \mathrm{g}^{-1}$ lipid, depending on the chemical. All of the measured concentrations were at least a factor of 3 above the quantification limit.

The concentrations of the analytes in the 4 fish sacrificed at the end of the uptake phase ranged from 0.3 to $14 \mu \mathrm{g} \mathrm{g}^{-1}$ lipid. The relative standard deviation of the concentration of a given analyte in the 4 fish ranged from 5 to $15 \%$, indicating that the interindividual variability was not large. The concentrations in the fish are provided in Table S2.

Determination of the Rate Constant for Overall Elimination $\left(k_{\mathrm{T}}\right)$. The rate constant for overall elimination $\left(k_{\mathrm{T}}\right)$ was determined from a regression of the natural logarithm of the concentration in the fish versus time for the eight fish analyzed, four of which were sacrificed at the beginning of the elimination experiment and four at the end $10 \mathrm{~d}$ later. The values ranged over 2 orders of magnitude, from 0.004 to $0.4 \mathrm{~d}^{-1}$ (see Table 1).

The uncertainty in $k_{\mathrm{T}}$, expressed as the relative standard error, ranged from $4 \%$ to $245 \%$ (see Table 1 ). There was a strong inverse correlation between the relative standard error and the magnitude of $k_{T}$ (see Figure S1). As the slope of the $C_{\text {Fish }}$ versus time relationship becomes shallower (i.e., as $k_{\mathrm{T}}$ gets smaller), the effect of the variability in $C_{\text {Fish }}$ becomes greater, leading to a higher uncertainty in the determination of $k_{\mathrm{T}}$. One approach to reducing this uncertainty would be to reduce the variability in $C_{\text {Fish }}$ by studying elimination kinetics in individual fish. This is currently hampered by the necessity to sacrifice a fish in order to determine $C_{\text {Fish }}$. However, with the development of in vivo passive sampling techniques for organic chemicals in fish, ${ }^{8-10}$ new options for studying uptake and elimination kinetics in individual fish are emerging.

Determination of the Rate Constant for Uptake $\left(k_{1}\right)$. The uptake rate constant was determined using the solution of the one compartment fish model with first order kinetics for fish exposed to a constant concentration of a chemical in water

$$
C_{\text {Fish }}=C_{\mathrm{W}} \frac{k_{1}}{k_{\mathrm{T}}}\left(1-\exp \left(-k_{\mathrm{T}} t\right)\right)
$$

where $t$ is the time for which the fish are exposed (here $4 \mathrm{~d}$ ). This equation was rearranged to yield

$$
k_{1}=\frac{C_{\text {Fish }}}{C_{\mathrm{W}}} k_{T} \frac{1}{\left(1-\exp \left(-k_{\mathrm{T}} t\right)\right)}
$$

$k_{1}$ was calculated using the mean values of $C_{\text {Fish }}$ for the 4 fish sacrificed at the end of the exposure period, the mean value of $C_{\mathrm{W}}$, and the $k_{\mathrm{T}}$ values determined above. The results are shown in Table 1 .

The uncertainty in the $k_{1}$ estimate is influenced by the variability in $C_{\text {Fish }}$ at the end of the exposure period. It is conceivable that this variability could be reduced by employing benchmarking, i.e., by measuring the accumulation of the test chemical relative to the accumulation of a benchmarking chemical. To explore this hypothesis, MUSK was chosen as a benchmarking chemical in analogy to the selection of a benchmark for BCF (see below), and $C_{\text {Fish }}$ was calculated as follows

$$
C_{\text {Fish,test }}=\overline{\left(\frac{C_{\text {Fish,test }}}{C_{\text {Fish,MUSK }}}\right)} C_{\text {Fish,MUSK }}
$$

The variability in the fresh weight normalized concentrations in the 4 fish was compared with the variability in the same concentrations normalized to the concentration of musk xylene (Figure 2). The results show that benchmarking markedly reduced the variability in the concentrations of ANI, PENT, PCB, HCB, and DDT (by a factor of 2-14), but that it increased the variability in the concentrations of ISBE, NP, and BUFE. An explanation for this can be found in the different

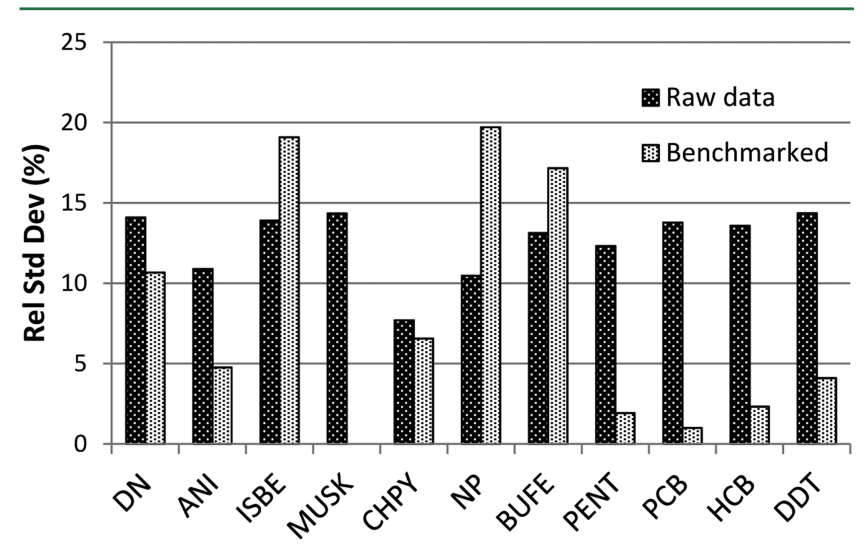

Figure 2. Variability of the wet weight normalized chemical concentration data in the 4 fish sacrificed at the end of the exposure phase, compared for the raw data and the data benchmarked to MUSK. 
processes governing the accumulation of the chemicals in the fish. For PENT, PCB, HCB, and DDT as well as for the benchmark MUSK, $k_{\mathrm{T}}$ was low and consequently the elimination of chemical during the uptake phase was negligible compared to the uptake. For these chemicals the variability in $C_{\text {Fish }}$ would have been governed by individual-specific differences in chemical uptake rate (e.g., ventilation). Such differences would be expected to affect the uptake of different chemicals in the same way, and hence benchmarking against one of these chemicals, MUSK, largely eliminated this source of variability. ISBE, NP, and ANI had $k_{\mathrm{T}}$ values that were sufficiently high that a significant portion $(>30 \%)$ of the chemical taken up during the uptake experiment was eliminated before the end of the exposure period. Other processes such as metabolism likely influenced this elimination. The interindividual variability in these processes need not be correlated with the interindividual variability in the chemical uptake rate. This can explain why benchmarking against MUSK did not reduce the variability in $C_{\text {Fish. }}$. This example illustrates that the use of benchmarking must be accompanied by an assessment of a) which sources of variability one is trying to eliminate and b) the potential of candidate benchmark chemicals to capture this variability.

Benchmarked $k_{1}$ values were calculated using both $C_{\text {Fish }}$ and $C_{\mathrm{W}}$ that had been benchmarked against MUSK

$$
k_{1 \text { test }}=\frac{\overline{C_{\text {Fish,test }} / C_{\text {Fish,MUSK }}}}{\overline{C_{\mathrm{W}, \text { test }} / C_{\text {W,MUSK }}}} k_{1 \text { MUSK }}
$$

These values differed from $k_{1}$ determined from nonbenchmarked data (Table 1) by at most $1.5 \%$. Thus, in this case benchmarking had little impact on the magnitude of the desired end point. It had a stronger impact on the uncertainty. The ratio of $k_{1}$ of the test chemical to $k_{1}$ of MUSK was less uncertain than the absolute value of $k_{1}$ for the same chemicals for which a reduction in uncertainty in $C_{\text {Fish }}$ was observed (ANI, PENT, PCB, HCB, and DDT). However, the magnitude of the reduction of uncertainty for $k_{1}(15-45 \%)$ was much less than the reduction in variability for $C_{\text {Fish }}$. This is because benchmarking did not reduce the variability in $C_{\mathrm{W}}$, and the variability in $C_{\mathrm{W}}$ made the dominant contribution to the uncertainty in $k_{1}$.

Determination of the Apparent Bioconcentration Factor $\left(\mathrm{BCF}_{\mathrm{A}}\right) \cdot \mathrm{BCF}_{\mathrm{A}}$ was determined by taking the quotient of $k_{1}$ and $k_{\mathrm{T}}$ and correcting to a lipid content of 5\%. In Table 1 the results are compared with the $\mathrm{BCF}_{\mathrm{A}}$ values measured in the parallel reference experiment conducted based on the OECD 305 guideline for bioconcentration in a flow-through fish test and reported elsewhere. ${ }^{6}$ The agreement is poor; the $\mathrm{BCF}_{\mathrm{A}}$ values from the abbreviated protocol are consistently lower by about a factor of 2 . The poor agreement is perhaps not surprising, given the much shorter test ( 2 weeks versus 12 weeks) and number of fish (8 versus 52).

To explore whether benchmarking could improve the performance of the abbreviated method, the $\mathrm{BCF}_{\mathrm{A}}$ results from this study were benchmarked against musk xylene according to

$$
\mathrm{BCF}_{\mathrm{BM}, \text { test }}=\mathrm{BCF}_{\text {Ref,MUSK }} \frac{\mathrm{BCF}_{\text {test }}}{\mathrm{BCF}_{\text {MUSK }}}
$$

Of the chemicals studied, musk xylene was judged to be the best choice for a benchmarking chemical because its bioconcentration was believed to be largely unaffected by metabolism (hence eliminating a possible source of variability in the BCF), it had an intermediate BCF that was reasonably close to regulatory threshold values, and the results from the reference experiment indicated that the measured value was among the most reliable of all chemicals studied.

The benchmarked $\mathrm{BCF}_{\mathrm{A}}$ values are compared with the values from the reference experiment in Table 1 . The difference was $<20 \%$ for 5 of the 9 test chemicals and $<30 \%$ for 7 of them. Poorer agreement was obtained for DDT and BUFE, two chemicals with very slow elimination rate constants and high $\mathrm{BCF}_{\mathrm{A}}$ values.

In the paper about the OECD 305-based reference experiment the BCF operating window of the methodology was discussed. It was concluded that there is an upper limit of the BCF value that can be measured in this kind of experiment. ${ }^{6}$ This limit is defined by the growth rate of the fish in the experiment. Referring to eq 4, as BCF increases and $k_{2}$ decreases, $k_{\mathrm{T}}$ and $k_{\mathrm{G}}$ become more similar in magnitude. Eventually these two measured parameters are not statistically distinguishable, and it is no longer possible to estimate $k_{2}$ or BCF. In this data set this was the case for DDT and BUFE. These chemicals were thus outside of the BCF operating window of the experiments.

For the 7 other chemicals that were within the BCF operating window of the experiments the agreement was good. The maximum discrepancy between the BBCF and reference experiments of $30 \%$ can be compared with independent measurements made with existing protocols. For instance, the MITI database contains two BCF determinations for CHPY that were conducted on the same species, yet they differed by a factor of 5.8 (493 versus 2880) (http://ambit.sourceforge.net/ euras/). This indicates that the $\mathrm{BBCF}$ protocol is a viable alternative to existing methods.

Determination of the Growth-Corrected Bioconcentration Factor $\left(\mathrm{BCF}_{\mathrm{No}-\mathrm{G}}\right)$. As discussed in the Theory section, growth corrected $\mathrm{BCF}$ values are desirable to facilitate the comparison of measured BCF values between studies and their extrapolation to the field. It was recently shown that internal benchmarking can also be used to measure $\mathrm{BCF}_{\mathrm{No}-\mathrm{G}}{ }^{6}$ In this case the benchmark is a persistent chemical with a value of $k_{2}$ that is so low that the fish's elimination of the chemical during the depuration experiment is negligible. The $k_{2}$ value of the test chemical can be calculated directly as the slope of a semilogarithmic plot of the quotient of the concentrations of the test chemical and the benchmark versus time (i.e., $\ln \left[C_{\text {Fish,test }} / C_{\text {Fish,bm }}\right]$ vs time)

$$
\begin{aligned}
k_{2} & =k_{\mathrm{T}}-k_{\mathrm{G}} \\
& =-\frac{\ln \left(C_{\text {Fish }, \text { tes }, t 2} / C_{\text {Fish }, \mathrm{bm}, t 2}\right)-\ln \left(C_{\text {Fish,tes }, t 1} / C_{\text {Fish }, \mathrm{bm}, t 1}\right)}{t_{2}-t_{1}}
\end{aligned}
$$

This principle was demonstrated using our results from the reference experiment with this mixture of chemicals. HCB was used as the growth dilution benchmark because $k_{\mathrm{T}}$ for HCB was not statistically different than the rate constant for lipid growth measured in that experiment $\left(0.0144 \mathrm{~d}^{-1}\right){ }^{6}$

To calculate $\mathrm{BCF}_{\mathrm{No}-\mathrm{G}}$ from the $\mathrm{BBCF}$ protocol, $\mathrm{HCB}$ was again used as the growth dilution benchmark as the $\mathrm{BBCF}$ and reference experiments were conducted in parallel using the same fish and food rations. The $k_{2}$ values were calculated as described above using the data for the eight fish analyzed, four of which were sacrificed at the beginning of the elimination 
Table 2. List of Test Chemicals That Fell within the BCF Operating Window of the Experiment Showing Their Elimination Rate Constant Excluding Growth Dilution $\left(k_{2}\right)$, Growth-Corrected Bioconcentration Factor $\left(\mathrm{BCF}_{\mathrm{No}-\mathrm{G}}\right)$ for the BBCF Experiment, $\mathrm{BCF}_{\mathrm{No}-\mathrm{G}}$ for the Reference Experiment, and Benchmarked $\mathrm{BCF}_{\mathrm{No}-\mathrm{G}}$ for the BBCF Experiment

\begin{tabular}{|c|c|c|c|c|c|c|}
\hline \multirow[b]{2}{*}{ chemical } & \multirow[b]{2}{*}{ abbrev } & \multicolumn{2}{|c|}{$k_{2}$} & \multicolumn{3}{|c|}{$\mathrm{BCF}_{\mathrm{No}-\mathrm{G}}\left(\mathrm{L} \mathrm{kg} \mathrm{ww}^{-1}, 5 \%\right.$ lipid $)$} \\
\hline & & value $\left(d^{-1}\right)$ & $\operatorname{RSE}^{a}(\%)$ & $\mathrm{BBCF}$ & reference & BBCF benchmarked \\
\hline 2,6-diisopropyl-naphthalene ${ }^{b}$ & DN & 0.076 & 8 & & & \\
\hline 2,3,4-trichloroanisole & ANI & 0.186 & 5 & 821 & 2269 & 1747 \\
\hline p-diisopropylbenzene & ISBE & 0.337 & 11 & 311 & 843 & 663 \\
\hline musk xylene & MUSK & 0.0158 & 11 & 13560 & 28861 & 28861 \\
\hline chlorpyrifos & CHPY & 0.117 & 16 & 1450 & 3583 & 3085 \\
\hline 4-n-nonylphenol & NP & 0.226 & 6 & 264 & 509 & 562 \\
\hline pentachlorobenzene & PENT & 0.0098 & 21 & 31508 & 89693 & 67059 \\
\hline 2,5-dichlorobiphenyl & РCB & 0.0075 & 31 & 101469 & 303351 & 215958 \\
\hline
\end{tabular}

${ }^{a}$ RSE stands for relative standard error. ${ }^{b}$ The concentrations of DN in water were not determined, so BCF could not be calculated.

experiment and four at the end $10 \mathrm{~d}$ later. The values are listed in Table 2 for the 7 chemicals that lay within the BCF operating window of the experiment. The $k_{2}$ values were used to calculate $\mathrm{BCF}_{\mathrm{No}-\mathrm{G}}$ according to eq 2 , and then $\mathrm{BCF}_{\mathrm{No}-\mathrm{G}}$ was benchmarked against MUSK

$$
\mathrm{BCF}_{\mathrm{No}-\mathrm{G}, \mathrm{BM}, \text { test }}=\mathrm{BCF}_{\mathrm{No}-\mathrm{G}, \text { Ref,MUSK }} \frac{\left(k_{1} / k_{2}\right)_{\mathrm{test}}}{\left(k_{1} / k_{2}\right)_{\mathrm{MUSK}}}
$$

Comparing these values with the values from the reference experiment (Table 2), one again sees that there is poor agreement between the nonbenchmarked $\mathrm{BCF}_{\mathrm{No}-\mathrm{G}}$ from the $\mathrm{BBCF}$ experiment and the corresponding values from the reference experiment, but that the benchmarked $\mathrm{BCF}_{\mathrm{No}-\mathrm{G}}$ values from the $\mathrm{BBCF}$ experiment agree well with the corresponding values from the reference experiment. This reaffirms the utility of the internal benchmarking of the BCF measurement identified above. It also illustrates how two benchmarks can be employed simultaneously for two distinct purposes.

Potential and Limitations of the BBCF Approach. Many of the advantages of the BBCF approach are obvious: a decrease in the number of fish required and the number of fish to be analyzed (excluding controls) by a factor of 5 and in the length of the experiment by up to a factor of 5. Further savings can be achieved by assessing several chemicals; the simultaneous exposure to 9 chemicals in this study reduced the number of fish required by an additional factor of 9 . The draft of a revised version of the OECD 305 guideline has pursued a similar strategy, proposing a "Minimised Aqueous Exposure Test" which prescribes two sample points during the uptake phase and two during the clearance phase (as opposed to one of each in this work). 5

While the shorter length of the experiment is one of the advantages of the BBCF approach, there could be grounds to extend the depuration phase of the experiment if one wished to expand the BCF operating window. The BCF operating window is defined by the growth rate of the fish. To exploit the full BCF operating window of the method, one must be able to quantify changes in the concentrations in the fish during the depuration experiment that arise from growth dilution only. For the growth rate of fish in this study, a depuration phase of $10 \mathrm{~d}$ was sufficient for this. To expand the BCF operating window it would be necessary to reduce the growth rate of the fish. To quantify changes in concentration due to growth only at a slower growth rate, a depuration phase longer than $10 \mathrm{~d}$ could be required.
The short length of the exposure phase could prove problematic for chemicals for which several days or more are required before biotransformation systems in the fish are activated. The assessment of such chemicals is particularly challenging, as one must ascertain whether activation occurs at the exposure levels that can be expected in the environment.

The BBCF approach is based on the hypothesis that the bioconcentration process can be described with a one box model employing first order kinetics. While this is true for many compounds, there are some chemicals which do not display first order kinetics. ANI in this study is one such example. A limitation of the BBCF protocol is that it does not allow an assessment of whether the bioconcentration adhered to first order kinetics, since concentrations are measured at the beginning and at the end of the depuration experiment. One way to overcome this limitation without sacrificing more fish is to use in vivo passive sampling. In vivo passive sampling using PDMS coated needles has been successfully employed to study the elimination kinetics of organic contaminants in fish. ${ }^{8-10}$

The BBCF approach is dependent on simultaneous exposure of the fish to at least two chemicals, namely the test chemical and the BCF benchmark. There is a risk that simultaneous exposure can influence the results for chemicals for which metabolism is a dominant elimination mechanism. If a second chemical either inhibits or induces enzymes involved in the metabolism of the test chemical, $k_{2}$ (and hence BCF) may be modified. A comparison of the $\mathrm{BCF}$ from the reference experiment in this study, which employed exposure to multiple chemicals, with BCFs from the literature determined in single chemical exposures yielded no evidence of such an effect. Nevertheless, chemicals that strongly effect metabolic systems should not be used as benchmarks, and caution should be exercised when testing chemicals for which metabolism is expected to be a dominant elimination pathway.

The major reason for the good performance of the BBCF protocol is the use of internal benchmarking. This is clearly illustrated by the comparison of the benchmarked and nonbenchmarked BCF values above. In addition to enabling large reductions in fish requirements and costs, internal benchmarking has the potential to markedly improve the interstudy comparability of BCF measurements. As outlined in the Introduction, large variability in the BCF values measured in different studies limits the utility of BCFs in a regulatory context. For instance, of the two measurements reported for CHPY above, one suggests that the chemical is 4 times below the REACH threshold for bioaccumulative chemicals (BCF = 2000), while the second measurement indicates that the 
chemical exceeds the threshold by almost 50\%. Internal benchmarking could reduce this variability, just as it reduced the differences between the results from the BBCF and reference experiments in this work. The draft of the revised version of the OECD 305 guideline does include a benchmarking element; the use of a reference compound is proposed as part of the dietary exposure test to control for food spiking technique together with the homogeneity and bioavailability of the chemical in the food. ${ }^{5}$ This method could profit from a much broader application of internal benchmarking, as demonstrated here.

Quantifying the BCF using internal benchmarking requires knowledge of the BCF value of the benchmark chemical. Given the uncertainty in $\mathrm{BCF}$ determination, obtaining consensus $\mathrm{BCF}$ values for benchmark chemicals to be used in regulatory contexts could be problematic. Ideally regulators should define a threshold chemical instead of a threshold BCF. The threshold chemical would be used as an internal benchmark in the BCF test. If the BCF of the chemical of interest exceeded the BCF of the internal benchmark, then the chemical would be flagged positive; if not it would be flagged negative. This measurement could be done with much better precision and accuracy than the existing BCF determination. Criteria for the selection of a threshold chemical (in addition to its bioconcentration properties) include low toxicity to fish, low tendency to induce or suppress metabolic systems in fish, stability in the aquaria system, and ease of analysis.

\section{ASSOCIATED CONTENT}

\section{S Supporting Information}

Four tables (sources of test chemicals and internal standards, the properties of the fish, the chemical concentration in the water during the uptake phase, and the chemical concentrations in the fish) and one figure (plot of the relative standard error in $k_{\mathrm{T}}$ versus $k_{\mathrm{T}}$ ). This material is available free of charge via the Internet at http://pubs.acs.org.

\section{AUTHOR INFORMATION}

\section{Corresponding Author}

*Phone: +46 8674 7228. E-mail: michael.mclachlan@itm.su.se.

\section{Notes}

The authors declare no competing financial interest.

\section{ACKNOWLEDGMENTS}

Kerstin Grunder is acknowledged for her excellent technical assistance. Matt MacLeod is thanked for helpful comments on the manuscript. We are grateful to the European Chemical Industry Council Long-Range Research Initiative for funding and to the research liaison team for useful discussions and helpful suggestions.

\section{REFERENCES}

(1) Organisation for Economic Co-operation and Development. OECD guidelines for testing of chemicals. Proposal for updating guideline 305. Bioconcentration: Flow-through fish test. Organisation for Economic Co-operation and Development (OECD), Paris, 1996.

(2) Van der Jagt, K.; Munn, S.; Tørsløv, J.; de Bruijn, J. Alternative approaches can reduce the use of test animals under REACH. Addendum to the report: Assessment of additional testing needs under REACH - Effects of (Q)SARS, risk based testing and voluntary industry initiatives. Report EUR 21405. European Commission, Joint Research Centre, Ispra, Italy, 2004.
(3) de Wolf, W.; Comber, M.; Douben, P.; Gimeno, S.; Holt, M.; Leonard, M.; Lillicrap, A.; Sijm, D.; van Egmond, R.; Whale, G. Animal use replacement, reduction, and refinement: Development of an integrated testing strategy for bioconcentration of chemicals in fish. Integr. Environ. Assess. Manage. 2007, 3, 3-17.

(4) Springer, T. A.; Guiney, P. D.; Krueger, H. O.; Jaber, M. J. Assessment of an approach to estimating aquatic bioconcentration factors using reduced sampling. Environ. Toxicol. Chem. 2008, 27, $2271-2280$

(5) Organisation for Economic Co-operation and Development. Test Guidelines Programme. Draft TG 305: Bioaccumulation in Fish: Aqueous and Dietary Exposure. Organisation for Economic Cooperation and Development (OECD), ENV/JM/TG(2012)31, Paris, 13 March 2012. http://www.oecd.org/dataoecd/10/48/50309198.pdf (accessed July 5, 2012).

(6) Adolfsson-Erici, M.; Åkerman, G.; McLachlan, M. S. Measuring bioconcentration factors in fish using exposure to multiple chemicals and internal benchmarking to correct for growth dilution. Environ. Toxicol. Chem. 2012, accepted.

(7) Adolfsson-Erici, M.; Åkerman, G.; Jahnke, A.; Mayer, P.; McLachlan, M. S. A flow-through passive dosing system for continuously supplying aqueous solutions of hydrophobic chemicals to bioconcentration and aquatic toxicity tests. Chemosphere 2011, 86, 593-599.

(8) Adolfsson-Erici, M.; Åkerman, G.; McLachlan, M. S. In-vivo passive sampling to measure elimination kinetics in bioaccumulation tests. Chemosphere 2012, 88, 62-68.

(9) Zhou, S. N.; Oakes, K. D.; Servos, M. R.; Pawliszyn, J. Application of solid-phase microextraction for in vivo laboratory and field sampling of pharmaceuticals in fish. Environ. Sci. Technol. 2008, 42, 6073-6079.

(10) Zhang, X.; Oakes, K. D.; Cui, S.; Bragg, L.; Servos, M. R.; Pawliszyn, J. Tissue-specific in vivo bioconcentration of pharmaceuticals in rainbow trout (Oncorhynchus mykiss) using space-resolved solid-phase microextraction. Environ. Sci. Technol. 2010, 44, 34173422 . 